\title{
RNA Regulators: Formidable Modulators of
}

\section{Yersinia Virulence}

\section{Aaron M. Nuss ${ }^{+}$, Ann Kathrin Heroven ${ }^{+}$and Petra Dersch*}

Department of Molecular Infection Biology, Helmholtz Centre for Infection Research, Braunschweig, Germany

*Corresponding author:

Petra Dersch

Dept. of Molecular Infection Biology,

Helmholtz Centre for Infection Research

38124 Braunschweig

Germany

Phone: +49-531-6181-5700

FAX: +49-531-6181-5709

e-mail: petra.dersch@helmholtz-hzi.de

+: These authors contributed equally to this work.

Keywords: Yersinia, regulatory RNAs, riboswitches, RNA thermometers, Csr, thermosensing, regulated RNA degradation, gene regulation, virulence 


\section{Summary}

A large repertoire of RNA-based regulatory mechanisms, including a plethora of cisand trans-acting non-coding RNAs (ncRNAs), sensory RNA elements, regulatory RNA-binding proteins and RNA degrading enzymes have been uncovered lately as key players in the regulation of metabolism, stress responses and virulence of the

genus Yersinia. Many of them are strictly controlled in response to fluctuating environmental conditions sensed during the course of the infection, and certain riboregulators have already been shown to be crucial for virulence. Some of them are highly conserved among the family of Enterobacteriaceae, while others are genus, species or strain-specific and could contribute to the difference in Yersinia pathogenicity. Importantly, the analysis of Yersinia riboregulators has not only uncovered crucial elements and regulatory mechanisms governing host-pathogen interactions, it also revealed exciting new venues for the design of novel anti-infectives. 


\section{Pathogenicity and Virulence Factors in Yersinia}

The three human pathogenic Yersinia species, Y. enterocolitica, Y. pseudotuberculosis and $Y$. pestis possess an arsenal of conserved and species-specific virulence factors that promote efficient colonization and persistence of the bacteria in mammals (humans, rodents, pigs/boars, goats, deer, and hares) and insect vectors (fleas). This repertoire enables Y. enterocolitica and Y. pseudotuberculosis (with 60-70\% genomic identity) to cause a range of similar, relatively benign, self-limiting intestinal diseases (yersiniosis). Y. pestis, which evolved clonally from Y. pseudotuberculosis two to ten thousand years ago (>97\% genomic identity), is the causative agent of plague $[1,2]$. Currently, the molecular mechanisms that determine the fundamental differences in the pathogenicity between both enteric Yersinia species and Y. pestis are unclear. Gene gain, loss and rearrangements have been considered to play an important role $[3,4]$. Several virulence factors such as the plasminogen activator Pla, the F1 capsular protein and Ymt (Yersinia murine toxin) have been found exclusively in $Y$. pestis, whereas the crucial adhesion and invasion factors of the enteric pathogens, invA and yadA, are inactivated [4]. This could account for their distinct difference in pathogenicity. In addition, there are variations within the regulatory networks controlling expression of virulence-related genes.

Up to now a large repertoire of conserved and species-specific riboregulators, including numerous cis- and trans-acting non-coding RNAs (ncRNAs), sensory RNA elements (RNA thermometers and riboswitches), regulatory RNA-binding proteins and RNA degrading enzymes, have been identified in pathogenic Yersiniae, and many of these riboregulators are part of large regulatory networks. They adjust the expression of colonization factors, toxins, host defense processes and virulencerelated physiological and metabolic processes in response to fluctuating environmental conditions encountered during the course of an infection (Figure 1, 
Table 1). This review summarizes the current state of knowledge regarding RNAmediated regulation in Yersinia. It describes the characteristics, function and regulation of all classes of Yersinia riboregulators with special emphasis on their role in pathogenesis.

\section{The Regulatory Non-coding RNA Repertoire of Yersinia which Impacts Virulence}

Regulatory ncRNAs are important players of the post-transcriptional regulation of virulence genes. They can interact with ribosomal binding sites or other 'complementary' sites within the coding region of a gene transcript. Such interactions can either modulate translation initiation and efficiency of the targeted mRNA and/or alter the access of RNases leading to an increased or decreased rate of mRNA decay.

\section{Trans-acting RNAs of Yersinia}

Among the first trans-acting small RNAs, which were shown to be important for the control of Yersinia virulence, were CsrB and CsrC [5]. Both ncRNAs and the dimeric RNA binding protein CsrA belong to the global carbon storage regulator system (Csr), which has been shown to control various metabolic, physiological and virulencerelated genes in response to environmental signals in other bacteria [6-8]. The CsrA protein binds to ANGGA motifs within 5'-untranslated regions (5'-UTRs) (i.e. overlapping the ribosome-binding site) or/and early coding regions of numerous target mRNAs. This leads to altered translation efficiency and transcript stability. CsrB and CsrC form a complex secondary structure with several stem loops with exposed single-stranded GGA motifs that bind and sequester multiple CsrA dimers, thus preventing its activity $[5,8]$. 
The Csr system affects a multitude of physiological and virulence-related activities in Yersinia. Initially it was shown that sequestration of CsrA by the Csr RNAs is crucial for the initiation of Y. pseudotuberculosis infection. Free CsrA controls expression of the two important colonization factors invasin (InvA) and PsaA through the RovM-RovA regulatory cascade $[5,9,10]$ (Figure 1). Deletion of csrA also abolishes the motility of Y. pseudotuberculosis and Y. enterocolitica, as CsrA is required for translation of the flagella master regulator flhDC $[5,11]$. Flagellar motility is required for efficient adhesion and subsequent invasion into host cells [12]. A microarray analysis further demonstrated that multiple genes of the virulence plasmid associated Ysc type III secretion system are differentially expressed in a csrA mutant of Y. pseudotuberculosis [13]. Furthermore, the Csr system is implicated in the control of biofilm formation, resistance against antibiotic, osmotic, and heat stress, as well as numerous metabolic pathways $[11,13,14]$. A major feature is that CsrA adjusts the central carbon flow through the pyruvate-acetate node and the tricarboxylic acid cycle in response to nutrient availability [13].

According to the myriad of influenced physiological activities, it is not surprising that the expression of the Csr system components is tightly regulated by multiple regulatory factors and environmental signals. In contrast to Escherichia coli and Salmonella Typhimurium, CsrB and CsrC of Yersinia are controlled by different two component systems, BarA/UvrY and PhoP/PhoQ (Figure 1). BarA/UvrY senses short chain fatty acids such as formate and acetate in $E$. coli to activate $\operatorname{csr} B[5,8,15]$, whereas the PhoP/PhoQ systems induces expression of the csrC gene, depending on the presence of divalent ions $\left(\mathrm{Mg}^{2+}, \mathrm{Mn}^{2+}\right.$, and $\left.\mathrm{Ca}^{2+}\right)$, secreted antimicrobial peptides and acidic $\mathrm{pH}$ [16]. PhoP-mediated activation results in transcription of two closely adjacent promoters, leading to two distinct CsrC RNAs. It is unknown whether the two transcripts have different regulatory properties. Furthermore, the cAMP 
receptor protein (Crp), a global regulator of ncRNA expression in Y. pseudotuberculosis [16], oppositely influences expression of the small RNAs CsrB and CsrC [17, 18] (Figure 1). This close interplay illustrates that Crp and CsrA are two key players of virulence management. A surprisingly large subset of growth phase responsive ncRNAs, in addition to Csr-type RNAs, was directly as well as indirectly regulated via Crp, which enables virulence and fitness control by ncRNA-based regulatory mechanisms according to the nutritional status of the bacteria $[13,16]$. Hence, it is reasonable that the replacement of costly proteinaceous regulatory elements by ncRNAs enhances fitness especially when nutrients are limited.

Several high-throughput RNA sequencing studies combined with sophisticated computational analyses have enabled the identification of more than 500 ncRNA candidates in Y. pestis (CO92, KIM6, and biovar Microtus) and Y. pseudotuberculosis (YPIII and IP32953) (Supplemental Table S1)[16, 19-24]. The majority of the Yersinia ncRNAs (mostly referred to as Ysr based on the initial analysis by Koo et al. [19]) were discovered in bacteria grown under different laboratory conditions. Unfortunately, parallel publications have resulted in the use of identical Ysr names for different ncRNAs from Y. pseudotuberculosis YPIII and Y. pestis CO92 [16, 24], for which we suggest an addition of the first two letters of the first author's surname to avoid confusion (Supplemental Table S1). Most identified ncRNAs are specific for Yersinia. This indicates that a large set of these riboregulators is exclusively involved in the fine-tuning of fitness during the different life-styles of this genus.

A thorough comparison of the identified ncRNA repertoires showed a surprisingly low overlap between the five different studies using Y. pestis (CO92, KIM6, and biovar Microtus) and Y. pseudotuberculosis (YPIII and IP32953) despite the overall high sequence conservation as well as the fact that sequences of almost all identified ncRNAs of these strains could be detected in the genome of IP32953 (Figure 1; 
Supplemental Table S1, $[16,25])$. Only the highly expressed and most conserved small RNAs were identified in most strains (e.g. MicA, GcvB, GImZ, Ffs, CyaR, CsrB, CsrC, GImY, and SsrS). This inconsistency is likely to be the result of different experimental conditions, distinct data analyses and different genome annotations. For instance, several trans-acting RNAs in one study (sR028, sR041, sR050, sR066 and sR070) identified in close proximity to adjacent ORFs [23] were reclassified as 5'-UTRs in another study [16]. Possibly, these sRNAs represent premature or processed transcripts of the 5'-UTR (Supplemental Table S1). Moreover, some validated ncRNAs were classified as trans-encoded RNAs in Y. pseudotuberculosis IP32953, but as antisense RNAs in Y. pseudotuberculosis YPIII, as they are encoded antisense to hypothetical proteins, which are not annotated in the IP32953 genome [16]. Furthermore, numerous ncRNAs are differentially expressed and/or contain point mutations, duplications (sibling RNAs), insertions/deletions altering their function and/or stability between and within the species [16, 19, 24, 26, 27]. For instance, the stability of the CsrC RNAs differs significantly between the Y. pseudotuberculosis YPIII and IP32953 due to a 20 nt insertion in $\operatorname{csrC}_{\mathrm{IP} 32953}$ [26]. This might partially explain present difficulties faced when attempting to draw conclusions about the presence, type, expression, stability and conservation of a particular ncRNA in the different human pathogenic Yersinia species. A more extensive analysis of multiple representative strains of the three human pathogenic Yersinia species under an identical experimental set-up and data analysis process is required to elucidate whether a certain identified ncRNA or a variant is specific for the distinct pathogenic properties.

Previous work has shown that the molecular RNA chaperone Hfq, which assists the function of many ncRNAs, is crucial for Yersinia pathogenesis [24, 28-31]. This indicates that ncRNAs contribute to Yersinia virulence, but which ncRNAs and to 
what extent they impact pathogenesis in the different Yersinia species is largely unknown. Several of the identified conserved ncRNAs (SgrS/Ysr150, MicF, MicA/Ysr7, GImY/Ysr147, GImZ/Ysr148, GcvB/Ysr45, YenS, SraG, FnrS, OmrAB, and Spot42), which are expressed under laboratory growth conditions, are likely to control important metabolic and stress adaptation genes [25, 32, 33]. They are involved in stress adaptation as well as in the outer membrane, cell wall, and iron and carbon metabolism in other Enterobacteriaceae, but their role for Yersinia virulence has not yet been investigated. So far, only one RNA-seq based approach was performed to profile the expression of Yersinia ncRNAs during host colonization. This study demonstrated that a small set of ncRNAs of a Y. pestis biovar Microtus strain was at least 4-fold more abundant during murine lung and spleen colonization compared to culture medium [23]. Among these ncRNAs were the Yersinia-specific ncRNAs sR039 as well as RyhB1, RyhB2, CyaR/RyeE and RybB, of which only RyhB1 and RyhB2 (Table 1) were further characterized. RyhB1 and RyhB2 abundance is highly increased during iron starvation and dependent on the ferricuptake regulator Fur [27, 34]. However, neither a ryhB1 nor a ryhB2 mutant was attenuated for virulence [27].

Deletion mutants of the conserved and six newly identified ncRNAs of $Y$. pestis biovar Microtus, which were found to be highly abundant in the lung and spleen (sR034, sR035/HmsB, sR055, sR073, sR84, and sR088), were used to infect mice either subcutaneously or intranasally [23]. However, most deletion mutants were not attenuated in virulence. This strongly suggests that these riboregulators fine-tune Yersinia fitness and virulence, and are part of robust regulatory networks in which other components can compensate for their function.

In the initial study performed by Koo et al. [19], mutants lacking Y. pseudotuberculosis ncRNAs Ysr23, Ysr29, and Ysr35 were tested for a virulence phenotype. In 
contrast to the $\Delta y s r 23$ strain, infections with the $\Delta y s r 29$ and the $\Delta y s r 35$ strains resulted in a significant difference in survival compared to the wild-type. A deletion of the ysr35 locus in $Y$. pestis also caused prolonged survival of mice following intranasal infection, indicating that Ysr35 may play a role in pathogenesis. Ysr35 is conserved between the two Yersinia species [19]. Its expression has been shown to increase during a thermal upshift supporting its role in virulence [35], but the molecular targets of this ncRNA are still unknown.

More is known about the Y. pseudotuberculosis-specific ncRNA Ysr29. A proteomic analysis for Ysr29 target identification revealed multiple proteins contributing to the general stress response as well as environmental and host stresses, including molecular chaperones (GroEL and DnaK), urease (UreC), 30S ribosomal protein S1 (RpsA), glutathione-S-transferase (Gst), peroxidase (AhpC) and the ribosome-recycling factor (Rrf) [19]. Transcript levels of the target genes were not affected by the lack of Ysr29, indicating that this ncRNA controls translation rather than mRNA stability.

A recent genome-wide analysis of ncRNAs expressed by $Y$. pestis further identified an unstable trans-acting RNA (Ysr141-SC) encoded upstream of the yopH gene of the virulence plasmid pCD1 (Supplemental Table S1). It enhances the synthesis of some type III secretion system (T3SS)-associated effector proteins, e.g. YopJ, YopE, YopK, YpkA and the T3SS/Yop activator LcrF, and reduces production of YopH most likely by altered transcript degradation and translation efficiency [24]. Ysr141-SC regulates YopJ production directly by base-pairing with the 5'-UTR of the yopJ transcript. Whether the change of YopJ levels is responsible for the dysregulation of the other T3SS/Yop components, or requires additional control factors is unclear.

\section{Antisense RNAs of Yersinia}


The genome-wide analysis of the Y. pseudotuberculosis YPIII transcriptome further identified 80 putative antisense RNAs of which 19 were encoded on the virulence plasmid pYV [16]. Included are multiple antisense RNAs (asRNAs), which are complementary to the transcripts of the effector YpkA, the V-antigen and several components of the T3SS machinery. This set constitutes a dense community compared to the lower number of antisense RNAs identified on the YPIII chromosome suggesting a fine-tuned control of T3SS/Yop expression. However, the precise molecular function and influence on pathogenesis of these plasmid-encoded asRNAs is yet unknown and much work remains to be done to characterize their influence on Yersinia biology.

A recent study demonstrated that $Y$. pseudotuberculosis upregulates the virulence-plasmid copy number during infection, which is indispensable for virulence [36]. Upregulation is caused by an increased expression of the IncFII plasmid replicase RepA, which is repressed under non-infection conditions by the antisense RNA CopA. A marked decrease in CopA levels results in a significant reduction of the ratio of the CopA antisense RNA and the repA transcript level in Y. pseudotuberculosis during the colonization of the Peyer's patches compared to bacteria grown in culture. How this regulatory tactic is induced during host tissue colonization is still unclear, but the translocon protein YopD was found to influence the copy-number increase [36].

A small number of asRNAs has also been identified in Y. pestis. One striking example is a transcript complementary to the plasminogen activator protease gene pla on the Y. pestis specific pPCP1 plasmid, which includes an ORF for a 48 aa peptide [37]. However, no physiological function has yet been assigned to this antipla transcript, and no equivalent peptide could be detected. Qu et al. identified the asRNAs Yp-sR3, Yp-sR7, Yp-sR8 and Yp-sR16/YsrH on the opposite strand of genes encoding for membrane proteins, the $50 \mathrm{~S}$ ribosomal protein and $\mathrm{FabH}$ an essential 
enzyme of fatty acid biosynthesis [21, 38], but their contribution to virulence is not known.

\section{Sensory RNAs in Yersinia}

Riboswitches and RNA thermometers belong to the class of cis-regulatory RNA elements with sensory functions and are typically located within 5'-UTRs. Both types of riboregulators influence downstream gene expression via structural alterations of the sensory RNA segment. While RNA thermometers function as thermo-controlled RNA zippers and typically affect mRNA translation, riboswitches usually alter gene expression on the transcriptional and post-transcriptional levels in response to ligand binding, i.e. binding of metabolites or metal ions.

\section{Riboswitches}

The current knowledge of riboswitches in Yersinia is rather scarce and is mainly based on a high-resolution whole genome transcriptomics study of Y. pseudotuberculosis in which numerous mRNAs with long 5'-UTRs (> $200 \mathrm{nt}$ ) were discovered [16]. A bioinformatics analysis predicted a few conserved riboswitches (Moco, FMN, cobalamin, yybP-ykoY element), the characterized $\mathrm{Mg}^{2+}$-responsive riboswitch $m g t A$ [39], and 17 additional riboswitch-like elements, indicating that the Yersinia riboswitch-repertoire still remains undiscovered. Recently, a powerful unbiased high-throughput approach for global discovery of riboswitches and attenuators (Term-seq) has been described [40]. The application of the Term-seq approach to Yersinia grown in different growth media or host environments will not only allow a rapid screening for riboregulators which respond to metabolites of choice, it will also enable the discovery of yet unknown virulence-related riboswitches. 


\section{RNA thermometers (RNATs)}

Temperature represents a major stimulus for Yersinia to adjust metabolism and virulence gene expression, indicating that besides proteineaceous thermosensors such as the virulence regulator RovA [41], RNATs also represent reasonable players in this process. Surprisingly, so far only one RNAT has been identified and characterized in Y. pseudotuberculosis, the IcrF RNAT located in the intergenic region of the $y s c W$ IcrF operon [42]. The transcriptional activator protein LcrF is crucial for the temperature-dependent induction of the virulence plasmid-encoded T3SS machinery and effector proteins. The IcrF RNAT consists of a two-stem loop in which the ribosomal binding site (RBS) is hidden in the second hairpin at $25^{\circ} \mathrm{C}$. The RBS becomes accessible at $37^{\circ} \mathrm{C}$ due to structural melting allowing ribosome binding and $\mathrm{lcrF}$ translation [42, 43]. As RNATs show little to poor sequence and structure conservation, bioinformatic predictions of novel RNATs are limited. However, a very recently performed global analysis of temperature-sensitive RNA structures of more than 1.750 RNAs of $Y$. pseudotuberculosis demonstrated a highly dynamic RNA structurome [44]. This analysis not only showed that reprogramming of the pathogen's virulence program and metabolism in response to temperature is associated with a large restructuring of numerous RNAs, it also led to the identification of 16 novel RNATs. Many of these RNATs are located within 5'-UTRs of transcripts related to virulence, the oxidative stress response, metabolism and heat shock (Figure 2) [44]. In addition to translation, the transcription of many RNAT-controlled genes (e.g. IcrF, ailA, $c n f Y, \operatorname{kat} A, \operatorname{sod} A, \operatorname{sod} B$ and $\operatorname{sod} C$ ) is also activated upon thermal upshift from moderate to host body temperature [16, 44]. This highlights the importance of a fine-tuned and multilayered control to rapidly adjust the pathogens' demands upon host entry. 


\section{Modification of Translation}

RNA binding proteins constitute another important class of riboregulators. They affect mRNA translation and turnover in diverse fashion, e.g. they can modulate ncRNAmRNA interactions and influence the access of the translational and RNA degradation machinery.

\section{Trans-translation by the SsrA-SmpB Tagging and Ribosome Rescue System}

The first RNA-based regulatory system shown to influence Yersinia virulence was the unique bacterial trans-translational control system, composed of the small stable RNA A (SsrA/tmRNA/10Sa/sR022/Yp-sR31) and the small RNA-binding protein B (SmpB) [45]. This system targets premature transcripts that lack stop codons to maintain the translational machinery of the bacteria operational by eliminating ribosomes that are stalled on the damaged/incomplete transcripts via transfer of the polypeptide chain to SsrA [46-48]. Notably, SsrA was among the ncRNAs, which were more abundant during murine lung and spleen colonization of $Y$. pestis biovar Microtus. An equivalent ssrA mutant strain was shown to be impaired in replication within macrophages and attenuated for virulence in mice [23]. This can be attributed to the fact that SsrA/SmpB influence (i) expression of the key virulence regulator LcrF, (ii) motility, and (iii) resistance towards oxidative and nitrosative stresses, and low $\mathrm{pH}[45,49]$.

\section{Regulatory RNA-binding Proteins}

In addition to the conserved ribosomal and translation proteins, the Yersinia-specific translocon protein YopD has a RNA-binding function [50, 51]. YopD (possibly alone or in complex with its secretion chaperone LcrH) was found to bind yop-encoding 
mRNAs in the 5'-UTRs reducing their translation either by an increase of yop mRNA degradation or hindering translation initiation [51]. The precise molecular mechanism is still unknown, but AU-rich regions in close proximity to the ribosome-binding site of the majority of yop mRNA seem important but alone are not sufficient for YopDmediated translational repression $[51,52]$. This suggests that other regulatory components contribute this process. In fact, the negative post-transcriptional regulator of the T3SS/Yop system LcrQ (YscM1 and YscM2 in Y. enterocolitica) was proposed to assist the YopD-LcrH complex [52]. Moreover, YopD was found to

interact with $30 \mathrm{~S}$ ribosomal particles [53], which suggests perturbation of $30 \mathrm{~S}$ complex formation and ribosome assembly on the ysc/yop mRNAs. A major future challenge will be to unravel the precise mechanism and the dynamic of this multicomponent and multilayered control process that allows the bacteria to carefully balance energy use for virulence factor production and biological fitness/growth.

\section{Changing mRNA Half-lives}

Continuous RNA decay is a prerequisite to enable adaptation to rapidly changing environmental conditions such as the ones experienced during the different stages of infection. Several RNA degrading enzymes are known to act cooperatively or independently. They recognize different target sites and degrade their targets with different efficiencies. Several factors such as RNA structure, protection by translating ribosomes and modification of the RNA (e.g. polyadenylation) determine the accessibility and degradation efficiency of these enzymes $[54,55]$. Based on the genome sequences all pathogenic Yersinia species encode multiple RNases (> 15). Among them are highly conserved endo- and exonucleases, such as polynucleotide phosphorylase (PNPase), RNase E, RNase G, RNase III and RNase Y/YbeY, which cleave internally or from the 3'-/5'-end of RNAs [56] (Table 1). They act on different 
pathways to mature rRNAs and tRNAs, process transcripts and intervene in the decay of numerous mRNAs. Together they form a global regulatory network, which is also crucial for the control of virulence gene expression. Despite the fact that these RNases are encoded by human pathogenic Yersiniae, not much is known about their implication in RNA-based control mechanisms that influence pathogenesis.

RNase E, a single-strand-specific endonuclease, and the exonuclease PNPase were shown to participate in virulence control. RNase $\mathrm{E}$ and PNPase are both required for optimal functioning of the Ysc/Yop T3SS [57-59]. Together with the enzyme enolase and RNA helicase B (RhIB) they form the Yersinia degradosome, which suggests that both control T3SS activity in a similar manner [60]. Since the levels of Ysc/Yop transcripts and proteins were increased or remained unchanged in the absence of a functional PNPase or RNase E, they seem to influence transcription/mRNA decay and activity of the T3SS by distinct mechanisms [61]. Moreover, the single-strand specific RNase $\mathrm{Y}$ (YbeY) that processes the 3 '-ends of the $16 \mathrm{~S}$ rRNA and is responsible for the late-stage $70 S$ ribosome quality control, also affects many virulence-related features, including acid stress resistance, cell adhesion/invasion properties and T3SS [62]. Among the YbeY-regulated ncRNAs are CsrB and CsrC of the global carbon starvation regulator system, which may explain the pleiotropic virulence-associated phenotypic changes of an ybe $Y$ mutant.

\section{Concluding Remarks}

Recent research led to the discovery of numerous riboregulators and RNA-based regulatory mechanisms evolved by Yersinia to control virulence and establish a successful infection. A continuously increasing set of ncRNAs and multiple sensory RNAs that are crucial for virulence have been identified through genome-wide screens. There are certainly more as the vast majority of identified ncRNAs have not 
yet been functionally characterized. A major future challenge will be to characterize their molecular targets and control mechanisms, the regulation of their expression and their role for pathogenicity. In addition, numerous intriguing questions and unaddressed issues associated with the Yersinia riboregulatory elements remain to be addressed. Some of them are highlighted in the Outstanding Questions.

We eagerly anticipate many exciting new insights into the role of species- and strain-specific riboregulator variants for pathogenesis. A detailed comparison of the identified ncRNAs in different strains of the human pathogenic Yersinia species showed that the composition and expression of the ncRNAs vary significantly between the closely related species Y. pestis and Y. pseudotuberculosis (Supplemental Table S1, [25]), suggesting that specific riboregulators or riboregulator variants could contribute to the pathological differences. However, several intra-species ncRNA variations (e.g. CsrC) or differences in the control of major regulators (e.g. Crp) have also been identified. They have a major influence on the expression of virulence factors [16, 26, 63], making the identification of these potential 'patho-specific' ncRNAs a major endeavour.

Moreover, a rapidly increasing knowledge about the participation of riboregulators in the control of Yersinia-host and insect vector interplay is expected, and some of them might represent well-suited drug targets and prone for the development of antiriboregulation strategies. Promising strategies are the selection of inhibitors that interfere with a central riboregulator such as CsrA [64], the design of tailor-made compounds, or RNAs that interfere with the essential LcrF thermometer, similar to the recently developed riboswitch inhibitors $[65,66]$.

Taken together, an impressive large repertoire of riboregulators has been recently discovered in the genus Yersinia, of which several have already been shown to be formidable modulators of virulence, although, our overall knowledge about their mole- 
cular targets, functions and role for pathogenesis is still in its infancy. The ongoing characterization of RNA-based control mechanism will not only open new avenues in our understanding of the control of host-pathogen interactions, it may also pave the way for the development of new anti-riboregulation strategies to combat infections.

\section{Acknowledgements}

We thank Martin Fenner for discussion, Whitney Weigel and Kwame Hagan for critical reading of the manuscript. German Research Foundation provided funding to Petra Dersch under the grant number DE616/4 for the analysis of sensory and regulatory RNAs of Yersinia. Petra Dersch is further supported by the German Center for Infection Research under grant number DZIF-TTU 06.801.

\section{References}

1. Achtman, M. et al. (1999) Yersinia pestis, the cause of plague, is a recently emerged clone of Yersinia pseudotuberculosis. Proc Natl Acad Sci U S A 96 (24), 14043-8.

2. Bos, K.I. et al. (2012) Yersinia pestis: new evidence for an old infection. PLoS One 7 (11), e49803.

3. Wren, B.W. (2003) The yersiniae--a model genus to study the rapid evolution of bacterial pathogens. Nature Reviews Microbiology 1 (1), 55-64.

4. McNally, A. et al. (2016) 'Add, stir and reduce': Yersinia spp. as model bacteria for pathogen evolution. Nat Rev Microbiol 14 (3), 177-90.

5. Heroven, A. et al. (2008) A Csr-type regulatory system, including small non-coding RNAs, regulates the global virulence regulator RovA of Yersinia pseudotuberculosis through RovM. Mol Microbiol 68 (5), 1179-95. 
6. Romeo, T. et al. (2013) Post-transcriptional regulation on a global scale: form and function of Csr/Rsm systems. Environ Microbiol 15 (2), 313-24.

7. Vakulskas, C.A. et al. (2015) Regulation of Bacterial Virulence by Csr (Rsm) Systems. Microbiol Mol Biol Rev 79 (2), 193-224.

8. Heroven, A.K. et al. (2012) The Csr/Rsm system of Yersinia and related pathogens: A post-transcriptional strategy for managing virulence. RNA Biol 9 (4).

9. Nagel, G. et al. (2001) Environmental control of invasin expression in Yersinia pseudotuberculosis is mediated by regulation of RovA, a transcriptional activator of the SlyA/Hor family. Mol Microbiol 41 (6), 1249-69.

10. Revell, P.A. and Miller, V.L. (2000) A chromosomally encoded regulator is required for expression of the Yersinia enterocolitica inv gene and for virulence. Mol Microbiol 35 (3), 677-85.

11. LeGrand, K. et al. (2015) CsrA impacts survival of Yersinia enterocolitica by affecting a myriad of physiological activities. BMC Microbiol 15, 31.

12. Young, G.M. et al. (2000) Motility is required to initiate host cell invasion by Yersinia enterocolitica. Infect Immun 68 (7), 4323-6.

13. Bücker, R. et al. (2014) The pyruvate-tricarboxylic acid cycle node: a focal point of virulence control in the enteric pathogen Yersinia pseudotuberculosis. J Biol Chem 289 (43), 30114-32.

14. Willias, S.P. et al. (2015) CRP-mediated carbon catabolite regulation of Yersinia pestis biofilm formation Is enhanced by the carbon storage regulator protein, CsrA. PLoS One 10 (8), e0135481.

15. Chavez, R.G. et al. (2010) The physiological stimulus for the BarA sensor kinase. J Bacteriol 192 (7), 2009-12. 
16. Nuss, A.M. et al. (2015) Transcriptomic profiling of Yersinia pseudotuberculosis reveals reprogramming of the Crp regulon by temperature and uncovers Crp as a master regulator of small RNAs. PLoS Genet 11 (3), e1005087.

17. Heroven, A.K. and Dersch, P. (2014) Coregulation of host-adapted metabolism and virulence by pathogenic yersiniae. Front Cell Infect Microbiol 4, 146.

18. Heroven, A.K. et al. (2012) Crp induces switching of the CsrB and CsrC RNAs in Yersinia pseudotuberculosis and links nutritional status to virulence. Front Cell Infect Microbiol 2, 158.

19. Koo, J.T. et al. (2011) Global discovery of small RNAs in Yersinia pseudotuberculosis identifies Yersinia-specific small, noncoding RNAs required for virulence. Proc Natl Acad Sci U S A 108 (37), E709-17.

20. Koo, J.T. and Lathem, W.W. (2012) Global discovery of small noncoding RNAs in pathogenic Yersinia species. Adv Exp Med Biol 954, 305-14.

21. Qu, Y. et al. (2012) Identification by cDNA cloning of abundant sRNAs in a human-avirulent Yersinia pestis strain grown under five different growth conditions. Future Microbiol 7 (4), 535-47.

22. Beauregard, A. et al. (2013) Identification and characterization of small RNAs in Yersinia pestis. RNA Biol 10 (3), 397-405.

23. Yan, Y. et al. (2013) Determination of sRNA expressions by RNA-seq in Yersinia pestis grown in vitro and during infection. PLoS One 8 (9), e74495.

24. Schiano, C.A. et al. (2014) Genome-wide analysis of small RNAs expressed by Yersinia pestis identifies a regulator of the Yop-Ysc type III secretion system. J Bacteriol 196 (9), 1659-70.

25. Martinez-Chavarria, L.C. and Vadyvaloo, V. (2015) Yersinia pestis and Yersinia pseudotuberculosis infection: a regulatory RNA perspective. Front Microbiol 6, 956. 
26. Nuss, A.M. et al. (2014) A direct link between the global regulator PhoP and the Csr regulon in Y. pseudotuberculosis through the small regulatory RNA CsrC. RNA Biol 11 (5), 580-93.

27. Deng, Z. et al. (2012) Two sRNA RyhB homologs from Yersinia pestis biovar microtus expressed in vivo have differential Hfq-dependent stability. Res Microbiol 163 (6-7), 413-8.

28. Schiano, C.A. et al. (2010) The small RNA chaperone Hfq is required for the virulence of Yersinia pseudotuberculosis. Infect Immun 78 (5), 2034-44.

29. Geng, J. et al. (2009) Involvement of the post-transcriptional regulator of Hfq in Yersinia pestis virulence. PLoS One 4 (7), e6213.

30. Kakoschke, T. et al. (2014) The RNA chaperone Hfq impacts growth, metabolism and production of virulence factors in Yersinia enterocolitica. PLoS One 9 (1), e86113.

31. Kakoschke, T.K. et al. (2016) The RNA chaperone Hfq is essential for virulence and modulates the expression of four adhesins in Yersinia enterocolitica. Sci Rep 6, 29275.

32. Papenfort, K. and Vogel, J. (2010) Regulatory RNA in bacterial pathogens. Cell Host Microbe 8 (1), 116-27.

33. Schiano, C.A. and Lathem, W.W. (2012) Post-transcriptional regulation of gene expression in Yersinia species. Front Cell Infect Microbiol 2, 129.

34. Deng, Z. et al. (2014) Rapid degradation of Hfq-free RyhB in Yersinia pestis by PNPase independent of putative ribonucleolytic complexes. Biomed Res Int 2014, 798918.

35. Shepherd, D.P. et al. (2013) Counting small RNA in pathogenic bacteria. Anal Chem 85 (10), 4938-43. 
36. Wang, H. et al. (2016) Increased plasmid copy number is essential for Yersinia T3SS function and virulence. Science 353 (6298), 492-5.

37. Sodeinde, O.A. and Goguen, J.D. (1989) Nucleotide sequence of the plasminogen activator gene of Yersinia pestis: relationship to ompT of Escherichia coli and gene E of Salmonella typhimurium. Infect Immun 57 (5), 1517-23.

38. Lu, P. et al. (2014) A cis-encoded sRNA controls the expression of fabH2 in Yersinia. FEBS Lett 588 (10), 1961-6.

39. Korth, M.M. and Sigel, R.K. (2012) Unusually high-affinity $M g(2+)$ binding at the AU-rich sequence within the antiterminator hairpin of a $\mathrm{Mg}(2+)$ riboswitch. Chem Biodivers 9 (9), 2035-49.

40. Dar, D. et al. (2016) Term-seq reveals abundant ribo-regulation of antibiotics resistance in bacteria. Science 352 (6282), aad9822.

41. Herbst, K. et al. (2009) Intrinsic thermal sensing controls proteolysis of Yersinia virulence regulator RovA. PLoS Pathog 5 (5), e1000435.

42. Böhme, K. et al. (2012) Concerted actions of a thermo-labile regulator and a unique intergenic RNA thermosensor control Yersinia virulence. PLoS Pathog 8 (2), e1002518.

43. Hoe, N.P. and Goguen, J.D. (1993) Temperature sensing in Yersinia pestis: translation of the LcrF activator protein is thermally regulated. J Bacteriol 175 (24), 7901-9.

44. Rhigetti, F. et al. (2016) Temperature-responsive in vitro RNA structurome of Yersinia pseudotuberculosis. Proc. Natl. Acad. Sci. 113(26):7237-42.

45. Okan, N.A. et al. (2006) A Role for the SmpB-SsrA system in Yersinia pseudotuberculosis pathogenesis. PLoS Pathog 2 (1), e6.

46. Keiler, K.C. (2008) Biology of trans-translation. Annu Rev Microbiol 62, 133-51. 
47. Karzai, A.W. et al. (2000) The SsrA-SmpB system for protein tagging, directed degradation and ribosome rescue. Nat Struct Biol 7 (6), 449-55.

48. Neubauer, C. et al. (2012) Decoding in the absence of a codon by tmRNA and SmpB in the ribosome. Science 335 (6074), 1366-9.

49. Okan, N.A. et al. (2010) The smpB-ssrA mutant of Yersinia pestis functions as a live attenuated vaccine to protect mice against pulmonary plague infection. Infect Immun 78 (3), 1284-93.

50. Anderson, D.M. et al. (2002) YopD and LcrH regulate expression of Yersinia enterocolitica YopQ by a posttranscriptional mechanism and bind to yopQ RNA. J Bacteriol 184 (5), 1287-95.

51. Chen, Y. and Anderson, D.M. (2011) Expression hierarchy in the Yersinia type III secretion system established through YopD recognition of RNA. Mol Microbiol 80 (4), 966-80.

52. Cambronne, E.D. and Schneewind, O. (2002) Yersinia enterocolitica type III secretion: yscM1 and yscM2 regulate yop gene expression by a posttranscriptional mechanism that targets the $5^{\prime}$ untranslated region of yop mRNA. J Bacteriol 184 (21), 5880-93.

53. Kopaskie, K.S. et al. (2013) Translational regulation of Yersinia enterocolitica mRNA encoding a type III secretion substrate. J Biol Chem 288 (49), 35478-88.

54. Deutscher, M.P. (2015) How bacterial cells keep ribonucleases under control. FEMS Microbiol Rev 39 (3), 350-61.

55. Hui, M.P. et al. (2014) Messenger RNA degradation in bacterial cells. Annu Rev Genet 48, 537-59.

56. Arraiano, C.M. et al. (2010) The critical role of RNA processing and degradation in the control of gene expression. FEMS Microbiol Rev 34 (5), 883-923. 
57. Yang, J. et al. (2008) RNase E regulates the Yersinia type 3 secretion system. J Bacteriol 190 (10), 3774-8.

58. Rosenzweig, J.A. et al. (2007) Polynucleotide phosphorylase independently controls virulence factor expression levels and export in Yersinia spp. FEMS Microbiol Lett 270 (2), 255-64.

59. Rosenzweig, J.A. et al. (2005) Modulation of Yersinia type three secretion system by the S1 domain of polynucleotide phosphorylase. J Biol Chem 280 (1), 156-63.

60. Rosenzweig, J.A. and Chopra, A.K. (2013) The exoribonuclease polynucleotide phosphorylase influences the virulence and stress responses of yersiniae and many other pathogens. Front Cell Infect Microbiol 3, 81.

61. Henry, A. et al. (2012) The Yersinia pseudotuberculosis degradosome is required for oxidative stress, while its PNPase subunit plays a degradosome-independent role in cold growth. FEMS Microbiol Lett 336 (2), 139-47.

62. Leskinen, K. et al. (2015) Absence of YbeY RNase compromises the growth and enhances the virulence plasmid gene expression of Yersinia enterocolitica $0: 3$. Microbiology 161 (Pt 2), 285-99.

63. Lathem, W.W. et al. (2014) Posttranscriptional regulation of the Yersinia pestis cyclic AMP receptor protein Crp and impact on virulence. MBio 5 (1), e01038-13.

64. Maurer, C.K. et al. (2016) Discovery of the first small-molecule CsrA-RNA interaction inhibitors using biophysical screening technologies. Future Med Chem $8(9), 931-47$.

65. Howe, J.A. et al. (2015) Selective small-molecule inhibition of an RNA structural element. Nature 526 (7575), 672-7.

66. Lee, C.H. et al. (2016) Therapeutic applications of aptamer-based riboswitches. Nucleic Acid Ther 26 (1), 44-51. 
67. Fang, N. et al. (2014) HmsB enhances biofilm formation in Yersinia pestis. Front Microbiol 5, 685.

68. Wang, H. et al. (2016) Increased plasmid copy-number is essential for Yersinia T3SS function and virulence. Science in press, Published Online 30 Jun 2016, DOI: 10.1126/science.aaf7501.

69. Schweer, J. et al. (2013) The cytotoxic necrotizing factor of Yersinia pseudotuberculosis (CNFY) enhances inflammation and Yop delivery during infection by activation of Rho GTPases. PLoS Pathog 9 (11), e1003746.

70. Schwiesow, L. et al. (2015) Yersinia type III secretion system master regulator LcrF. J Bacteriol 198 (4), 604-14.

71. Bellows, L.E. et al. (2012) Hfq-dependent, co-ordinate control of cyclic diguanylate synthesis and catabolism in the plague pathogen Yersinia pestis. Mol Microbiol 86 (3), 661-74.

72. Williams, A.W. and Straley, S.C. (1998) YopD of Yersinia pestis plays a role in negative regulation of the low- calcium response in addition to its role in translocation of Yops. J Bacteriol 180 (2), 350-8. 


\section{Figure Legends}

Figure 1. Regulatory Network of Virulence Factors Controlled by Small ncRNAs in Yersinia. Overview of regulatory and environmental factors that control expression of virulence-associated traits in Yersinia including host colonization, host-adapted metabolism and immune defense. Transcriptional and post-transcriptional effects and involved ncRNAs (in yellow) are listed.

Figure 2. RNA Thermometers of Yersinia. Overview of the identified RNA thermometers of Y. pseudotuberculosis controlling virulence, oxidative stress, heat shock and metabolic functions. These all prevent translation of the target mRNAs at $25^{\circ} \mathrm{C}$, but not at $37^{\circ} \mathrm{C}$, due to incorporation of the ribosome binding site into a thermo-sensitive stem-loop structure. Genes given in red have previously been shown to be temperature-regulated. 